\title{
Studies on the Effect of Various Sterilization Procedures for in vitro Propagation of Carnation (Dianthus caryophyllus L.)
}

\author{
Bharti Gautam*, Puja Sharma, Y.C. Gupta, Anil Handa, \\ Manisha Thakur and Priyanka Sharma
}

Department of Floriculture and Landscape Architecture, Dr Y.S.Parmar University of Horticulture and Forestry, Nauni, Solan (HP)-173230, India

*Corresponding author

\section{A B S T R A C T}

\section{Keywords}

In vitro, Surface sterilization, Heavy metals, Hazardous, Uncontaminated growing cultures

Article Info

Accepted:

07 January 2019

Available Online:

10 February 2019
The present investigation entitled, "Studies on the effect of various sterilization procedures for in vitro propagation Of Carnation (Dianthus caryophyllus L.) was carried out at the Plant Tissue Culture Laboratory of Department of Floriculture and Landscape Architecture, Dr Y S Parmar University of Horticulture and Forestry, Nauni, Solan (HP) as a refinement in already existing protocol to find suitable and less hazardous surface sterilization chemicals than Mercuric Chloride which is one of the most widely used surface sterilant in micropropagation of carnation. The use of this chemical is being prohibited because of the presence of heavy metal ions in it, causing environment hazards. The necessity to consider an alternative surface sterilization agent is therefore obvious. The experiments were laid out in a Completely Randomized Design (factorial) consisting of two cultivars i.e. 'Parendillo' and 'Yellow Star'. Treatment of explants with $5 \%$ Calcium Hypochlorite is suggested as a potential substitute for Mercuric Chloride. $100 \%$ uncontaminated growing cultures in cv. 'Parendillo' and 'Yellow Star' were obtained with $5 \%$ Calcium Hypochlorite treatment of explants for 10 and 15 minutes, respectively.

\section{Introduction}

Carnation (Dianthus caryophyllus L.) is one of the most important cut flower crops in the world. The global cut flower market is maintained by the introduction of the new improved cultivars. Classical breeding has long been the main route for generation of new traits into a wide range of the commercial cultivars. The most successful and most widely used discipline of plant tissue culture technique is micropropagation which refers to the propagation of plants by using meristem tip culture which is the transfer of apical buds and surrounding leaf primordia to sterile culture conditions. It is one of the major floriculture crops in many countries of the world with high ornamental and commercial value (Burchi et al., 1996). Surface sterilization of explants is the basic step to ensure uncontaminated growing cultures. Different chemicals are being used for this purpose worldwide. One of the most widely used chemical is Mercuric Chloride $\left(\mathrm{HgCl}_{2}\right)$. This chemical, is however, is being prohibited because of presence of heavy metal ions in it (Fakhrfeshani et al., 2012) causing environment hazards. Environmental side 
effects of Mercuric Chloride have also been reported by Counter and Buchanan, 2004. The necessity to consider an alternative surface sterilization agent is therefore obvious. A refinement in already existing protocol is, therefore, required to find out a potential surface sterilization chemical which could be used as an alternative to Mercuric Chloride.

\section{Materials and Methods}

\section{Cleaning and sterilization of instruments and glasswares}

All the glasswares used for the experimentation were cleaned in a solution of $10 \%(\mathrm{v} / \mathrm{v})$ teepolprior to use. In addition, ultra violet light was kept on for half an hour in a laminar air flow cabinet and air flow was allowed for at least 10-15 minutes after putting off the ultra violet light. All the instruments and other accessories were wrapped carefully in aluminium foil and wet sterilized by steam in an autoclave. All the instruments were wiped with ethyl alcohol before use.

\section{Preparation of culture medium}

In vitro studies were conducted on Murashige and Skoog (1962) nutrient medium. For convenience sake and in order to avoid weighing individual ingredients each time, concentrated stock solutions of macro elements, micro elements and vitamins were prepared and stored in refrigerator. All the stock solutions were mixed in a small amount of distilled water to prepare a required medium. The final volume was made by adding distilled water and by supplementing with $3 \%$ sucrose and the $\mathrm{pH}$ was adjusted to 5.8 with $1 \mathrm{~N} \mathrm{HCl}$ or $1 \mathrm{~N} \mathrm{NaOH}$. Agar-Agar $(0.65 \%)$ was dissolved by heating the medium. The hot medium was poured into the test tubes which were plugged with nonabsorbent cotton plugs and were sterilized in an autoclave at $121^{\circ} \mathrm{C}$ and $1.05 \mathrm{Kg} / \mathrm{cm}^{2}$ pressure (15 psi) for 20 minutes (Dodds and Roberts, 1982). The medium was then kept at room temperature and used for culturing after 2 days waiting period to check for any contamination in the medium.

\section{Surface sterilization of explants}

In order to study the effect of surface sterilants on per cent uncontaminated growing cultures in carnation surface sterilants viz. 0.1 $\%$ Mercuric Chloride (one concentration), 3\% Sodium Hypochlorite and 5\% Calcium Hypochlorite were used at different concentrations and varying durations to obtain contamination free cultures (shoot and nodal explants). The cultures were established on MS Medium consisting of $2 \mathrm{ppm}$ BA. The design used was Completely Randomized Design (Factorial). Total number of uncontaminated growing cultures out of total cultures was counted after one month of culturing and per cent was calculated. The details of the experiment were as follows:

Explants: Two (Apical and Nodal)

Cultivars: Two (Parendillo and Yellow Star)

Surface Sterilants: Three

\section{Treatments}

$0.1 \%$ Mercuric Chloride for 3 minutes

$3 \%$ Sodium Hypochlorite for 5 minutes

$3 \%$ Sodium Hypochlorite for 10 minutes

$3 \%$ Sodium Hypochlorite for 15 minutes

$3 \%$ Sodium Hypochlorite for 20 minutes

$3 \%$ Sodium Hypochlorite for 25 minutes

$3 \%$ Sodium Hypochlorite for 30 minutes

$3 \%$ Sodium Hypochlorite for 35 minutes

$5 \%$ Calcium Hypochlorite for 5 minutes

$5 \%$ Calcium Hypochlorite for 10 minutes

$5 \%$ Calcium Hypochlorite for 15 minutes

$5 \%$ Calcium Hypochlorite for 20 minutes

$5 \%$ Calcium Hypochlorite for 25 minutes

$5 \%$ Calcium Hypochlorite for 30 minutes

$5 \%$ Calcium Hypochlorite for 35 minutes 
Treatments: 15

Treatment combinations: $\quad 2 \times 2 \times 15=60$

Number of replications:

$$
3
$$

\section{Results and Discussion}

The results in the Table 1 shows that there was a variable difference among the cultivars with respect to per cent uncontaminated growing cultures with more per cent uncontaminated cultures found in cv. 'Parendillo' than 'Yellow Star'. It could be attributed to the genotypic differences among the cultivars. Varietal difference was also observed by Dharma (2003) while working with carnation cultivars 'Tempo' and 'Diplomat'.

Table.1 Effect of surface sterilization treatments and explant source on percent uncontaminated growing cultures (4 weeks after inoculation)

\begin{tabular}{|c|c|c|c|c|c|c|}
\hline \multirow{2}{*}{\multicolumn{2}{|c|}{$\begin{array}{l}\text { Surface sterilization } \\
\text { Treatments (min.) }\end{array}$}} & \multicolumn{2}{|c|}{ Cultivars of carnation } & \multirow{3}{*}{$\begin{array}{l}\text { Mean } \\
93.75 \\
(76.94)\end{array}$} & \multicolumn{2}{|l|}{ Explants } \\
\hline & & \multirow{2}{*}{$\begin{array}{l}\text { Parendillo } \\
95.83 \\
(80.47)^{*}\end{array}$} & \multirow{2}{*}{$\begin{array}{l}\text { Yellow Star } \\
91.67 \\
(73.40)\end{array}$} & & \multirow{2}{*}{$\begin{array}{l}\text { Shoot tip } \\
95.83 \\
(80.47)\end{array}$} & \multirow{2}{*}{$\begin{array}{l}\text { Nodal } \\
91.67 \\
(73.40)\end{array}$} \\
\hline $\mathrm{HgCl}_{2}(0.1 \%)$ & 3 & & & & & \\
\hline \multirow[t]{7}{*}{$\mathrm{NaOCl}(3 \%)$} & 5 & $\begin{array}{l}22.67 \\
(28.29)\end{array}$ & $\begin{array}{l}25.00 \\
(29.87)\end{array}$ & $\begin{array}{l}23.83 \\
(29.08)\end{array}$ & $\begin{array}{l}23.33 \\
(28.76)\end{array}$ & $\begin{array}{l}24.33 \\
(29.08)\end{array}$ \\
\hline & 10 & $\begin{array}{l}54.00 \\
(47.32)\end{array}$ & $\begin{array}{l}48.33 \\
(44.03)\end{array}$ & $\begin{array}{l}51.17 \\
(45.68)\end{array}$ & $\begin{array}{l}56.67 \\
(48.85)\end{array}$ & $\begin{array}{l}45.67 \\
(42.50)\end{array}$ \\
\hline & 15 & $\begin{array}{l}85.00 \\
(67.29)\end{array}$ & $\begin{array}{l}84.17 \\
(66.68)\end{array}$ & $\begin{array}{l}84.58 \\
(66.98)\end{array}$ & $\begin{array}{l}83.33 \\
(65.95)\end{array}$ & $\begin{array}{l}85.83 \\
(68.01)\end{array}$ \\
\hline & 20 & $\begin{array}{l}77.67 \\
(61.82)\end{array}$ & $\begin{array}{l}71.67 \\
(57.98)\end{array}$ & $\begin{array}{l}74.67 \\
(59.90)\end{array}$ & $\begin{array}{l}71.67 \\
(57.95)\end{array}$ & $\begin{array}{l}77.67 \\
(61.86) \\
\end{array}$ \\
\hline & 25 & $\begin{array}{l}69.33 \\
(56.43)\end{array}$ & $\begin{array}{l}67.17 \\
(55.11)\end{array}$ & $\begin{array}{l}\mathbf{6 8 . 2 5} \\
(55.77)\end{array}$ & $\begin{array}{l}69.67 \\
(56.65)\end{array}$ & $\begin{array}{l}66.83 \\
(54.89)\end{array}$ \\
\hline & 30 & $\begin{array}{l}64.67 \\
(53.55)\end{array}$ & $\begin{array}{l}51.67 \\
(45.96)\end{array}$ & $\begin{array}{l}\mathbf{5 8 . 1 7} \\
(\mathbf{4 9 . 7 5 )}\end{array}$ & $\begin{array}{l}60.00 \\
(50.84)\end{array}$ & $\begin{array}{l}56.33 \\
(48.67)\end{array}$ \\
\hline & 35 & $\begin{array}{l}59.00 \\
(50.21)\end{array}$ & $\begin{array}{l}45.83 \\
(42.60)\end{array}$ & $\begin{array}{l}52.42 \\
(46.41)\end{array}$ & $\begin{array}{l}55.00 \\
(47.92)\end{array}$ & $\begin{array}{l}49.83 \\
(44.90)\end{array}$ \\
\hline \multirow[t]{7}{*}{$\mathrm{CaOCl}(5 \%)$} & 5 & $\begin{array}{l}92.50 \\
(76.99)\end{array}$ & $\begin{array}{l}78.33 \\
(64.89)\end{array}$ & $\begin{array}{l}85.42 \\
(70.94)\end{array}$ & $\begin{array}{l}80.00 \\
(66.25)\end{array}$ & $\begin{array}{l}90.83 \\
(75.63)\end{array}$ \\
\hline & 10 & $\begin{array}{l}100.00 \\
(90.00)\end{array}$ & $\begin{array}{l}85.00 \\
(69.27)\end{array}$ & $\begin{array}{l}92.50 \\
(79.64)\end{array}$ & $\begin{array}{l}90.00 \\
(76.77)\end{array}$ & $\begin{array}{l}95.00 \\
(82.50)\end{array}$ \\
\hline & 15 & $\begin{array}{l}100.00 \\
(90.00)\end{array}$ & $\begin{array}{l}100.00 \\
(90.00)\end{array}$ & $\begin{array}{l}100.00 \\
(90.00)\end{array}$ & $\begin{array}{l}100.00 \\
(90.00)\end{array}$ & $\begin{array}{l}100.00 \\
(90.00)\end{array}$ \\
\hline & 20 & $\begin{array}{l}100.00 \\
(90.00)\end{array}$ & $\begin{array}{l}100.00 \\
(90.00)\end{array}$ & $\begin{array}{l}100.00 \\
(90.00)\end{array}$ & $\begin{array}{l}100.00 \\
(90.00)\end{array}$ & $\begin{array}{l}100.00 \\
(90.00)\end{array}$ \\
\hline & 25 & $\begin{array}{l}100.00 \\
(90.00)\end{array}$ & $\begin{array}{l}100.00 \\
(90.00)\end{array}$ & $\begin{array}{l}100.00 \\
(90.00)\end{array}$ & $\begin{array}{l}100.00 \\
(90.00)\end{array}$ & $\begin{array}{l}100.00 \\
(90.00)\end{array}$ \\
\hline & 30 & $\begin{array}{l}89.17 \\
(72.75)\end{array}$ & $\begin{array}{l}95.00 \\
(82.50)\end{array}$ & $\begin{array}{l}92.08 \\
(77.63)\end{array}$ & $\begin{array}{l}91.67 \\
(76.55)\end{array}$ & $\begin{array}{l}92.50 \\
(78.70)\end{array}$ \\
\hline & 35 & $\begin{array}{l}84.17 \\
(62.77)\end{array}$ & $\begin{array}{l}93.33 \\
(79.43)\end{array}$ & $\begin{array}{l}88.75 \\
(73.10) \\
\end{array}$ & $\begin{array}{l}88.33 \\
(71.93)\end{array}$ & $\begin{array}{l}89.17 \\
(74.27) \\
\end{array}$ \\
\hline \multicolumn{2}{|l|}{ Mean } & $\begin{array}{l}79.60 \\
(68.13)\end{array}$ & $\begin{array}{l}75.81 \\
(65.45)\end{array}$ & - & $\begin{array}{l}77.70 \\
(66.59)\end{array}$ & $\begin{array}{l}77.71 \\
(66.98)\end{array}$ \\
\hline \multirow{2}{*}{\multicolumn{2}{|c|}{$\begin{array}{l}\text { Explants } \\
\text { Shoot tip }\end{array}$}} & & & $\mathrm{CD}_{0.05^{\mathrm{f}}}$ & & \\
\hline & & $\begin{array}{l}81.00 \\
(69.41)\end{array}$ & $\begin{array}{l}74.40 \\
(63.78)\end{array}$ & \multicolumn{2}{|c|}{$\begin{array}{l}\text { Cultivars } \\
\text { Explants } \\
\text { Treatments } \\
\text { Cultivars x Treatments } \\
\text { Explants x x Treatments } \\
\text { Cultivars x Explant }\end{array}$} & $\begin{array}{l}1.52 \\
\text { NS } \\
4.17 \\
5.90 \\
5.90 \\
2.16\end{array}$ \\
\hline
\end{tabular}

\footnotetext{
*values in parenthesis are arc sine transformed values
} 
Table.2 Interaction effect of cultivars, surface sterilization treatments and explant source on percent uncontaminated growing cultures (4 weeks after inoculation)

\begin{tabular}{|c|c|c|c|c|c|}
\hline \multirow{2}{*}{\multicolumn{2}{|c|}{$\begin{array}{l}\text { Surface } \quad \text { sterilization } \\
\text { Treatments (min.) }\end{array}$}} & \multicolumn{4}{|c|}{ Cultivars of carnation } \\
\hline & & \multicolumn{2}{|c|}{ Parendillo } & \multicolumn{2}{|c|}{ Yellow Star } \\
\hline & & Shoot tip & Nodal & Shoot tip & Nodal \\
\hline $\begin{array}{l}\mathrm{HgCl}_{2} \\
(0.1 \%)\end{array}$ & 3 & $\begin{array}{l}98.33 \\
(85.69)^{*}\end{array}$ & $\begin{array}{l}93.33 \\
(75.24)\end{array}$ & $\begin{array}{l}93.33 \\
(75.24)\end{array}$ & $\begin{array}{l}90.00 \\
(71.57)\end{array}$ \\
\hline \multirow[t]{7}{*}{$\mathrm{NaOCl}(3 \%)$} & 5 & $\begin{array}{l}20.00 \\
(26.45)\end{array}$ & $\begin{array}{l}25.33 \\
(30.12)\end{array}$ & $\begin{array}{l}26.67 \\
(31.07)\end{array}$ & $\begin{array}{l}23.33 \\
(28.67)\end{array}$ \\
\hline & 10 & $\begin{array}{l}60.00 \\
(50.79)\end{array}$ & $\begin{array}{l}48.00 \\
(43.85)\end{array}$ & $\begin{array}{l}53.33 \\
(46.91)\end{array}$ & $\begin{array}{l}43.33 \\
(41.15)\end{array}$ \\
\hline & 15 & $\begin{array}{l}83.33 \\
(65.95)\end{array}$ & $\begin{array}{l}86.67 \\
(68.62)\end{array}$ & $\begin{array}{l}83.33 \\
(65.95)\end{array}$ & $\begin{array}{l}85.00 \\
(67.40)\end{array}$ \\
\hline & 20 & $\begin{array}{l}76.67 \\
(61.15)\end{array}$ & $\begin{array}{l}78.67 \\
(62.50)\end{array}$ & $\begin{array}{l}66.67 \\
(54.75)\end{array}$ & $\begin{array}{l}76.67 \\
(61.21)\end{array}$ \\
\hline & 25 & $\begin{array}{l}73.33 \\
(58.93)\end{array}$ & $\begin{array}{l}65.33 \\
(53.93)\end{array}$ & $\begin{array}{l}66.00 \\
(54.37)\end{array}$ & $\begin{array}{l}68.33 \\
(55.85)\end{array}$ \\
\hline & 30 & $\begin{array}{l}66.67 \\
(54.75)\end{array}$ & $\begin{array}{l}62.67 \\
(52.34)\end{array}$ & $\begin{array}{l}53.33 \\
(46.92)\end{array}$ & $\begin{array}{l}50.00 \\
(45.00)\end{array}$ \\
\hline & 35 & $\begin{array}{l}63.33 \\
(52.74)\end{array}$ & $\begin{array}{l}54.67 \\
(47.68)\end{array}$ & $\begin{array}{l}46.67 \\
(43.09)\end{array}$ & $\begin{array}{l}45.00 \\
(42.12)\end{array}$ \\
\hline \multirow[t]{7}{*}{$\mathrm{CaOCl}(5 \%)$} & 5 & $\begin{array}{l}93.33 \\
(77.71)\end{array}$ & $\begin{array}{l}91.67 \\
(76.26)\end{array}$ & $\begin{array}{l}66.67 \\
(54.78)\end{array}$ & $\begin{array}{l}90.00 \\
(75.00)\end{array}$ \\
\hline & 10 & $\begin{array}{l}100.00 \\
(90.00)\end{array}$ & $\begin{array}{l}100.00 \\
(90.00)\end{array}$ & $\begin{array}{l}80.00 \\
(63.55)\end{array}$ & $\begin{array}{l}90.00 \\
(75.00)\end{array}$ \\
\hline & 15 & $\begin{array}{l}100.00 \\
(90.00)\end{array}$ & $\begin{array}{l}100.00 \\
(90.00)\end{array}$ & $\begin{array}{l}100.00 \\
(90.00)\end{array}$ & $\begin{array}{l}100.00 \\
(90.00)\end{array}$ \\
\hline & 20 & $\begin{array}{l}100.00 \\
(90.00)\end{array}$ & $\begin{array}{l}100.00 \\
(90.00)\end{array}$ & $\begin{array}{l}100.00 \\
(90.00)\end{array}$ & $\begin{array}{l}100.00 \\
(90.00)\end{array}$ \\
\hline & 25 & $\begin{array}{l}100.00 \\
(90.00)\end{array}$ & $\begin{array}{l}100.00 \\
(90.00)\end{array}$ & $\begin{array}{l}100.00 \\
(90.00)\end{array}$ & $\begin{array}{l}100.00 \\
(90.00)\end{array}$ \\
\hline & 30 & $\begin{array}{l}93.33 \\
(78.09)\end{array}$ & $\begin{array}{l}85.00 \\
(67.41)\end{array}$ & $\begin{array}{l}90.00 \\
(75.00)\end{array}$ & $\begin{array}{l}100.00 \\
(90.00)\end{array}$ \\
\hline & 35 & $\begin{array}{l}86.67 \\
(68.86)\end{array}$ & $\begin{array}{l}81.67 \\
(64.69)\end{array}$ & $\begin{array}{l}90.00 \\
(75.00)\end{array}$ & $\begin{array}{l}96.67 \\
(83.86)\end{array}$ \\
\hline
\end{tabular}

*values in parenthesis are arc sine transformed values

$\mathrm{CD}_{0.05}$ for: Cultivars x Treatments x Explants: 8.35

Among the different chemicals used for surface sterilization, Calcium Hypochlorite was found to be the most superior resulting in maximum uncontamination as compared to Mercuric Chloride and Sodium Hypochlorite doses.

$100 \%$ uncontaminated growing cultures were obtained when explants of cv. 'Parendillo' were surface sterilized with $5 \%$ Calcium
Hypochlorite $(\mathrm{CaOCl})$ for 10, 15, 20 and 25 minutes irrespective of explants source (Table 1 and 2). On the other hand, similar results in 'Yellow Star' were recorded with $5 \%$ Calcium Hypochlorite $(\mathrm{CaOCl})$ for 15, 20, 25 and 30 minutes.

Use of Calcium Hypochlorite $(\mathrm{CaOCl})$ for surface sterilization of explants has been reported by many workers. Sangwan et al., 
(1987) successfully used $5 \%$ Calcium Hypochlorite $(\mathrm{CaOCl})$ for surface sterilization of carnation shoots for 10 minutes.

Similarly, Roest and Bokelmann (1981) carried out surface sterilization of flower pedicels of carnation with $5 \%$ Calcium Hypochlorite $(\mathrm{CaOCl})$ for 20 minutes. The results obtained with Mercuric Chloride used for 3 minutes were, however, at par with uncontaminated cultures obtained from shoot tip explants of cv. 'Parendillo'.

Our findings suggest that the use of $5 \%$ Calcium Hypochlorite $(\mathrm{CaOCl})$ for 15,20 , and 25 minutes as surface sterilization treatment which gives better results i.e. $100 \%$ uncontaminated growing cultures over Mercuric Chloride. Therefore, it could be suggested as the potential alternative to Mercuric Chloride for surface sterilization.

\section{References}

Burchi G, Mecuri A De Benedetti L and Giovannini A. 1996. Transformation methods applicable to ornamental plants. Plant Tissue Culture Biotechnology, 2: 94-104

Counter S A and Buchanan L H. 2004. Mercury exposure in children: a review.
Toxicology and Applied Pharmacology, 198: 209-230

Dharma S. 2003. Studies on factors influencing the production of hardened glaucous carnation plantlets. M.Sc. Thesis, submitted to Dr. Y S Parmar University of Horticulture and Forestry, Nauni, Solan (H.P.).

Dodds J H and Roberts L W. 1982. Experiments in Plant Tissue Culture. Cambridge University Press, London, $178 \mathrm{pp}$.

Fakhrfeshani M, Bagheri A and Sharifi A. 2012. Disinfecting effects of nano silver fluids in Gerbera (Gerbera jamesonii) capitulum tissue culture. J. Biol. Environ. Sci., 6(17): 121-127

Murashige T and Skoog F. 1962. A revised medium for rapid growth and bioassay with tobacco tissue cultures. Plant Physiology, 15: 472-497

Roest S and Bokelmann G S. 1981. Vegetative propagation of carnation in vitro through multiple shoot development. Scientia Horticulturae, 14: 357-366

Sangwan R S, Detrez C and Sangwan N B S. 1987. In vitro culture of shoot tip meristems in some higher plants. ActaHorticulturae, 212(11): 661-666

\section{How to cite this article:}

Bharti Gautam, Puja Sharma, Y.C. Gupta, Anil Handa, Manisha Thakur and Priyanka Sharma. 2019. Studies on the Effect of Various Sterilization Procedures for in vitro Propagation of Carnation (Dianthus caryophyllus L.). Int.J.Curr.Microbiol.App.Sci. 8(02): 481-485. doi: https://doi.org/10.20546/ijcmas.2019.802.054 